In der Rubrik „Literatur kompakt" werden die wichtigsten Originalarbeiten aus der internationalen Fachliteratur referiert.

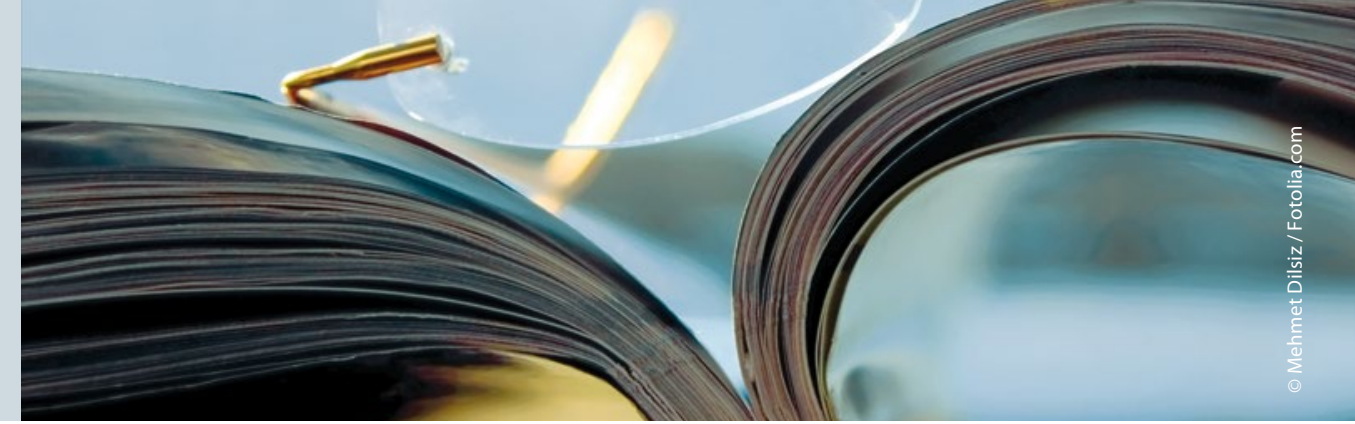

\section{Niedrigrisiko-Prostatakarzinom: nach Operation bessere Prognose als vermutet}

\begin{abstract}
Männer, die an einem Niedrigrisiko-Prostatakarzinom erkrankt sind, haben selbst dann ein geringeres Metastasierungs- und Sterberisiko als Patienten mit Intermediär- oder Hochrisikokarzinomen, wenn zum Zeitpunkt der Prostatektomie ungünstige pathologische Faktoren wie extrakapsuläres Wachstum entdeckt werden.
\end{abstract}

W elchen Einfluss präoperative Risikofaktoren auf die Entwicklung von Metastasen und auf die krebsspezifische Mortalität haben, überprüften Hamburger Urologen um Katharina Böhm vom Universitätsklinikum Hamburg-Eppendorf und der Universitätsmedizin Mainz anhand von Daten, die an der Martini-Klinik Hamburg zwischen 1992 und 2008 erhoben worden waren. Von den fast 7.000 Patienten mit radikaler Prostatektomie hatten 3.261 ein Niedrigrisiko-Karzinom und 3.682 ein Intermediär- oder Hochrisikokarzinom.

Untersucht wurden das Risiko für ein biochemisches Rezidiv, für die Entstehung von Metastasen sowie die krebsspezifische Mortalität zehn Jahre nach dem Eingriff, und zwar bei Vorliegen ungünstiger pathologischer Faktoren. Ihr Augenmerk richteten die Urologen dabei auf das extrakapsuläre Wachstum, Gleason-Score $\geq 3+4$ sowie $\geq 4+3$, positive Schnittränder, positive Lymphknoten und Samenblaseninvasion. Das mediane Follow-up betrug 96 Monate.

Wie die Ärzte berichten, lag bei extrakapsulärem Wachstum nach zehn Jahren der Anteil der Männer mit einem biochemischen Rezidiv in der NiedrigrisikoGruppe im Vergleich zu Männern mit Intermediär- oder Hochrisikotumoren bei
$26 \%$ versus $50 \%$. Die geschätzte ZehnJahres-Mortalität betrug $2 \%$ versus $5 \%$, die Metastasierungsrate $1,6 \%$ versus $8 \%$. Zum Zeitpunkt der abschließenden histologischen Untersuchung erreichte bei Männern mit einem Gleason-Score $\geq 3+4$ die Rate der Patienten mit einem biochemischen Rezidiv $25 \%$ versus $48 \%$ und bei denjenigen Männern mit einer Metastasierung nach zehn Jahren $3 \%$ versus $12 \%$. Die krebsspezifische Sterberate lag in dieser Gruppe bei $3 \%$ versus $8 \%$.

Schließlich ermittelten die Urologen um Böhm bei Patienten mit einem Gleason-Score $\geq 4+3$ nach zehn Jahren eine Rate für biochemische Rezidive von $56 \%$ versus $68 \%$, eine Metastasierungsrate von $11 \%$ versus $24 \%$ und eine krebsspezifische Mortalität von $6 \%$ versus $17 \%$. Alle Unterschiede - außer bei der Mortalität in der Gruppe der Männer mit hohem Gleason-Score - waren signifikant. Das galt auch für den Risikofaktor „positive Schnittränder", der mit einer biochemischen Rezidivrate nach zehn Jahren von $32 \%$ versus $66 \%$ und mit einer Metastasierungsrate von 2,9\% versus $15 \%$ sowie einer krebsspezifischen Mortalität von $4 \%$ versus $10 \%$ assoziiert war.

Aus der Multivariatanalyse, in der unter anderem das Alter der Patienten, das Jahr des chirurgischen Eingriffs, der
Lymphknotenstatus sowie der GleasonScore berücksichtigt wurden, geht hervor, dass der Niedrigrisiko-Status ein prädiktiver Faktor für die Metastasierung ist, und zwar bei Männern mit einem pathologischen Gleason-Score $\geq 3+4$. In diesem Fall ist die Wahrscheinlichkeit für die Tumorzellstreuung im Vergleich zu Männern mit Intermediär- oder Hochrisikotumoren um 49 \% niedriger (Hazard Ratio [HR]: 0,51; 95\%-Konfidenzintervall zwischen 0,33 und 0,$78 ; p=0,002$ ). Bei Männern mit Gleason-Score $\geq 4+3$ errechneten die Urologen eine HR von 0,41 (95\%-Konfidenzintervall zwischen $0,17$ und 0,$99 ; p=0,047)$. Ähnlich niedrig lag der Wert bei Vorliegen positiver Schnittränder (HR: 0,46; $95 \%$-Konfidenzintervall zwischen 0,22 und 0,97; $p=0,041)$. Schließlich errechneten die Hamburger Urologen für die krebsspezifische Mortalität bei Männern mit einem Gleason-Score $\geq 3+4$ eine HR von 0,62 (95\%-Konfidenzintervall zwischen 0,41 und 0,$93 ; \mathrm{p}=0,021$ ).

Fazit: Nach Ansicht von Böhm und ihren Kollegen sollten die Studienergebnisse bei der Beratung von Niedrigrisiko-Patienten genutzt werden, bei denen während der Prostatektomie ein Upgrading und/oder ein Upstaging erfolgt. Ihnen müsse vermittelt werden, dass ihre Prognose noch immer nicht so schlecht ist wie bei Patienten mit Intermediär-oder Hochrisikotumoren mit ungünstigen pathologischen Faktoren.

Peter Leiner

Böhm K et al. Impact of preoperative risk on metastatic progression and cancer specific death in patients with adverse pathology at radical prostatectomy. BJU Int. 2017; doi: 10.1111/ bju.13887. 